\title{
Clinical research monitoring: scenarios and challenges
}

\author{
Gustavo Adolfo Sierra Romero[ ${ }^{[1],[2]}$
}

[1]. Núcleo de Medicina Tropical, Campus Universitário Darcy Ribeiro, Universidade de Brasília, Brasília, DF. [2]. Instituto de Avaliação de Tecnologias em Saúde - IATS/CNPq, Porto Alegre, RS.

\begin{abstract}
Clinical research is essential for the development of new drugs, diagnostic tests and new devices. Clinical monitoring is implemented to improve the quality of research and attain high ethical and scientific standards. This review discusses the role of clinical monitors, taking into account the variety of scenarios in which medical research is developed, and highlights the challenges faced by research teams to ensure that patients rights are respected and that the social role of scientific research is preserved. Specific emphasis is given to the ethical dilemmas related to the multiple roles which clinical monitors play in the research framework, mainly those involving the delicate equilibrium between the loyalty to the sponsor and to the research subjects. The essential role of clinical monitoring for research developed in poor healthcare scenarios is highlighted as an approach to get the local infrastructure strengthening needed to achieve an adequate level of good clinical practices.
\end{abstract}

Keywords: Clinical research. Clinical monitoring. Clinical trials. Ethics. Good clinical practices.

\section{INTRODUCTION}

Clinical research is becoming an essential activity for the rational development of new pharmacological treatments, diagnostic tests and devices such as prostheses. The clinical research performed to substantiate the indication for new drugs, however, absorbs most of the efforts in this field ${ }^{1}$. The process for conducting research of adequate quality consumes a significant amount of the financial resources of the for-profit pharmaceutical industry, public agencies and, more recently, the non-profit, private organizations that assume the task of stimulating drug development for neglected diseases such as tuberculosis, sleeping sickness and leishmaniasis, among others ${ }^{2}$.

\section{RESEARCH SCENARIOS}

Regardless of the funding source for the development of clinical studies, the standardization of the appropriate conduct for studies is essential for trial success. Thus, the process has been established by the International Conference on Harmonisation through several recommendations that summarize the definitions, processes and functions of each professional involved in research. The goal of these recommendations is to standardize the criteria used in drug development to facilitate registration with the responsible agencies in the United States, Europe and Japan and to gather as much information as possible regarding experiments around the world $\mathrm{d}^{3-6}$.

Certainly, there are different scenarios for clinical research that entail various levels of vulnerability for the different agents involved. Research volunteers have different socioeconomic conditions that directly impact their ability to consent to

Address to: Dr. Gustavo Adolfo Sierra Romero. Núcleo de Medicina Tropical/UnB. Campus Universitário Darcy Ribeiro, Caixa Postal 04517, 70904-970 Brasília, DF, Brasil.

Phone: 5561 3107-1843

e-mail: gromero@unb.br

Received in 02/08/2012

Accepted in 08/10/2012 participate, and they are protected by legal frameworks that vary across countries and states. This vulnerability has been the subject of concern for more than five decades and the subject of discussion in the Declaration of Helsinki, a document that enshrines the basic principles of protection for study participants $^{7-11}$.

The challenge of conducting biomedical research in a variety of scenarios is directly related to the need for universal ethical principles in a multicultural world that exhibits multiplicities of health care systems with significant differences in healthcare standards ${ }^{12-13}$.

\section{THE NEED FOR RESEARCH MONITORING}

Clinical monitoring emerged from the need to monitor all processes related to study preparation, research implementation, research execution and procedures for the closure of field activities.

Concrete monitoring objectives include the following: protecting the rights and welfare of research participants; ensuring that the trial reports are complete and verifiable from source documents (previously agreed upon when planning the study); ensuring that the study is conducted according to the study protocol and the official modifications to the implementation process; and following good clinical practice and meeting all of the requirements of the applicable rules ${ }^{3}$. It is clear that monitors have two major dimensions to their activities: the ethical dimension related to protecting the research subjects and the technical dimension related to supervising specific activities during the research procedures. The need to ensure that these two dimensions are appropriately handled while developing studies imposes the need for monitoring. Thus, the monitor is an important agent in biomedical research.

\section{MONITOR PROFILES AND THEIR INTEGRATION IN RESEARCH}

Traditionally, it is recommended that the monitor be a professional who is properly qualified for the tasks of monitoring 
and who possesses a thorough knowledge of good clinical practices to ensure an accurate adherence to research procedures and the implementation of appropriate corrective measures when there are deviations from the planned trial ${ }^{3}$. It is also essential that the monitor has a comprehensive knowledge of formal and informal ethical issues regarding the performance of biomedical research.

Thus, the first question regarding the role of the monitor involves their clinical integration and subordination to the other agents involved in the research including the project sponsor, the clinical investigator, the committee responsible for monitoring data on efficacy and safety ${ }^{14,15}$, the research ethics committee ${ }^{16,17}$ and the government regulatory agencies responsible for registering new drugs.

Regardless of the funding source for the research to be monitored, the monitor is usually appointed by the research sponsor to whom the monitor should be loyal. The principles that guide the monitor's actions are within the general framework of good clinical practice. However, the extent and specific nature of the activities undertaken by the monitor are subject to the sponsor's discretion. This discretionary monitoring is directly related to the assumption that different scenarios require different intensities and qualities of monitoring.

Considering the possibility that the monitor can act as a relentless watchdog of research activities and lose sight of the essential purpose of guaranteeing trial quality, it is beneficial to undergo a training process that promotes creative problem solving and enhances good clinical practices, including good laboratory practices, and the quality of the clinical laboratories that perform the research.

In addition, the monitor can be a professional who transforms situations that require improvements in the infrastructure and in reception and clinical care to make the situation suitable. This point is extremely relevant when considering the needs to develop drugs or diagnostic tests for neglected diseases because often the trial volunteers are treated at health facilities that are in poor condition. In this situation, the effort to achieve the appropriate standard of care to perform the studies should include the monitor's active participation, especially during visits made before the trial begins.

From the moment the monitor is directly involved in the challenging task of helping to establish the conditions necessary for research and assumes the role of communicator and of the interpreter of local needs to the sponsor, there may be a shift, at least partially, of their loyalty from the sponsor toward a more basic and essential loyalty, the loyalty to the volunteers participating in the research, without which the monitor's mission loses meaning.

The above aspects are important to avoid the risk of establishing a relationship based on mistrust between the sponsor and the investigator mediated by the monitor without losing monitor objectivity, thus contextualizing the relationships between sponsors, monitors and researchers in a sphere of confidence where the goals are the protection of participants and the excellence of scientific evidence to be obtained through research.

\section{SPECIFIC DUTIES OF CLINICAL MONITORS}

The description of the specific tasks in the process of monitoring clinical studies has been published in detail ${ }^{3}$. This review will emphasize, in addition to the main task of serving as the most important means of communication between the sponsor and the investigator, those tasks that directly ensure that the welfare and rights of research subjects are respected and that the quality of the data obtained is preserved.

It falls to the monitor to verify that the research team is qualified to perform the study and that its composition and training remain adequate throughout the research period. The monitor's responsibility extends to tasks relating to the supply, supervision and disposal of the research materials, including the drug or the diagnostic test being studied and all relevant documentation for the investigator.

However, the monitor's most important task is to ensure that the research is executed according to the protocol so that the rights and safety of subjects are guaranteed. Thus, the monitor's participation is critical during an adverse reaction report to support the researchers according to the plan outlined in the protocol.

The monitor's role as evaluator of the conditions surrounding the invitation of potential candidates to participate as research subjects, the conditions of the site for obtaining the informed consent form and the characteristics of the procedures for signing the consent form is also relevant and should be carefully reviewed during the monitor's visit before starting the research. However, the most effort-consuming task is the review of the data collection forms for each subject to check the consistency of the information against the original documents that are used as original sources.

The activity of the monitors is usually summarized in reports submitted to the sponsor that include details of the activities performed during the monitoring visits, including the approaches adopted and those suggested to the researcher to correct or prevent potential problems.

\section{THE ETHICAL ASSUMPTIONS OF MONITOR ACTIONS AND THE RELATIONSHP TO THE RESEARCH ETHICS COMMITTEE}

The following applies to the practice of clinical monitoring: all ethical principles that guide the activities of the researchers involved in the clinical studies; the Declaration of Helsinki; and all applicable international, national and institutional rules where research is performed. Although the clinical monitor does not have the direct responsibility of complying with the recommendations of all of these dispositions, it is up to the monitor to ensure that they are faithfully followed during the study. Therefore, the monitor becomes ethically and morally co-responsible for the actions taken. Although the sponsor and the investigator are legally responsible for the research and this responsibility appears to carry more relevant or larger direct implications, the monitor holds some moral responsibility. It is understood that the monitor's role needs to be considered by the monitor and other agents involved in research. 
As the quality of the data obtained by the research depends directly and indirectly on the quality of monitoring, there is also the co-responsibility for what can happen when knowledge is disseminated.

Considering that currently, the use of any drug, diagnostic test or medical device depends on the quality of the scientific evidence that supports such use, the quality of monitoring will clearly be linked to the potential use that may be recommended from the results.

In the context of encouraging greater monitor participation from the early stages of implementing the studies, it is possible to consider the potential role of the monitor as a co-author of the articles to be published from the results of the monitored tests. By taking into account the concepts related to scientific authorship described by the International Committee of Medical Journal Editors ${ }^{18}$, it is suggested that the authors meet at least three conditions: first, a substantial contribution to the study's concept and design, data collection, analysis or interpretation; second, drafting the article or critical revision of the article's intellectual content; and third, approval of the final version to be published. Then, monitors do not strictly qualify as co-authors of a study. However, the crucial contribution of monitors to the development of studies should be recognized by name in the description of collaborations to prevent the monitor's participation from remaining anonymous, thus reducing the risk of discouraging the professionalization of monitoring by means of public recognition in the academic environment.

The relationship between the monitor and the research ethics committee does not happen directly because the researcher and the sponsor assign the monitor. However, it falls to the monitor to quickly report any deviation or imminent risk to the research volunteers and to suggest that the investigator or sponsor inform the committee of any facts that are considered relevant. In this sense, the management of situations related mainly to the occurrence of serious adverse events is crucial, and the monitor must ensure that the information regarding these events can be transmitted to the sponsor as quickly as possible. The responsibility of the monitor for the group monitoring data on safety and efficacy is clearly indirect, and it is up to the sponsor to guarantee the flow of information that feeds group discussions and guides decision-making, especially when the early termination of a clinical trial is considered ${ }^{14}$.

\section{MODELS FOR MONITORING, CERTIFICATION AND PROFESSIONALIZATION}

There are several models for the implementation of clinical monitoring. The most common is the monitoring of research contracted by a sponsor, or a so-called Contract Research Organization (CRO), and the financial recognition agreements for each activity are individually agreed upon with each monitor. The requirements for certification as a clinical monitor also vary depending on the sponsor, but there are organizations dedicated to this process. These organizations fall within the category of paid clinical monitors and become a source of consultation for recruiting monitors by the sponsors. The Brazilian Society of
Professionals in Clinical Research has developed activities to improve and disseminate knowledge on this field and maintains a certification program for clinical monitors. The Tropical Diseases, Special Programme for Research and Training (TDR) initiative for monitoring the clinical trials of drug development for neglected diseases has the peculiarity of working with the system for training monitors who already have an employment relationship with other institutions and perform clinical monitoring ad honorem when working on projects supported by TDR. The training of monitors by TDR has been performed by the program itself; however, recently, the demand for an independent certifier has increased for both the program and the monitors. The biggest challenge now is implementing clinical monitoring for publicly funded research projects that are not given resources to pay for professional monitoring and depend, therefore, on ad honorem monitoring from partnerships with other institutions such as TDR or on non-certified monitors. Certainly, the spread of good clinical practice between the critical mass of researchers can facilitate the monitoring capacity for this situation. It can be argued that clinical monitoring is a profession established within the framework of clinical research and that, regardless of the funding model, follows the general principles of action that have improved the quality of clinical trials to make them more reliable and safe.

\section{POSSIBILITIES FOR CONSTRUCTING NEW PRACTICE SCENARIOS}

The appropriation of concepts and practices of good clinical practice by clinical research teams has a collateral impact on other research groups that perform epidemiological research. Often, researchers who perform clinical trials also perform epidemiological studies to identify risk factors for diseases. Therefore, the principles of good practice are easily transferred to the practice of epidemiological research, which also involves ethical dimensions and techniques that should be monitored to ensure the safety and welfare of research subjects and the quality of specific procedures. Healthcare in programs for the supervised treatment of diseases such as tuberculosis have shown that the monitoring tasks have flexibility and a potential for the appropriation of practices and specific responsibilities for each scenario where the diseases occur ${ }^{19}$. Likewise, the monitoring tasks could be performed by different agents depending on the type of study, the vulnerability and the local capacity for appropriating the concepts and practices that are relevant for high quality monitoring.

In conclusion, clinical monitoring is currently an essential activity to ensure the safety and welfare of research subjects and simultaneously improves the reliability of the data obtained and has an enormous potential for application, representing a constant challenge for its balanced implementation in the context of the existing relationships between sponsors, research institutions and researchers.

\section{CONFLICT OF INTEREST}

The author declare that there is no conflict of interest. 


\section{REFERENCES}

1. Pocock SJ. The historical development of clinical trials. In: Pocock SJ, editor. Clinical Trials. Chichester: John Willey \& Sons; 1987. p. 14-27.

2. Drug for neglected diseases initiative. 2011 Annual report [Internet]. [Cited 2012 July 20]. Available from: http://www.dndi.org/images/stories/annual_ report/2011/DNDi_Annual\%20report\%202011_low-res.pdf/.

3. International Conference on harmonisation of technical requirements for registration of pharmaceuticals for human use. ICH harmonised tripartite guideline. Guideline for good clinical practice E6(R1) [Internet]. [Cited 2012 August 2]. Available from: http://www.ich.org/fileadmin/Public Web Site/ ICH_Products/Guidelines/Efficacy/E6_R1/Step4/E6_R1_Guideline.pdf/.

4. International Conference on harmonisation of technical requirements for registration of pharmaceuticals for human use. $\mathrm{ICH}$ harmonised tripartite guideline. General considerations for clinical trials E8. [Internet] [Cited 2012 August 2]. Available from: http://www.ich.org/fileadmin/Public Web_Site/ ICH_Products/Guidelines/Efficacy/E8/Step4/E8_Guideline.pdf.

5. International Conference on harmonisation of technical requirements for registration of pharmaceuticals for human use. ICH harmonised tripartite guideline. Choice of control group and related issues in clinical trials E10. [Internet] [Cited 2012 August 2]. http://www.ich.org/fileadmin/Public_Web_ Site/ICH_Products/Guidelines/Efficacy/E10/Step4/E10_Guideline.pdf.

6. International Conference on harmonisation of technical requirements for registration of pharmaceuticals for human use. $\mathrm{ICH}$ harmonised tripartite guideline. Statistical guidelines for clinical trials E9. [Internet] [Cited 2012 August 2]. Available from: http://www.ich.org/fileadmin/Public_Web_Site/ ICH_Products/Guidelines/Efficacy/E9/Step4/E9_Guideline.pdf.

7. World Medical Association. Policy. World Medical Association Declaration of Helsinki. Ethical principles for medical research involving human subjects. [Internet] [Cited 2012 August 2]. Available from: http://www.wma.net/ en/30publications/10policies/b3/.

8. World Medical Association. Declaration of Helsinki: Recommendations guiding physicians in biomedical research involving human subjects. JAMA $1997 ; 277: 925-926$
9. Romero GAS. Ensaios clínicos: reflexões éticas. In: Guilhem D, Zicker F, orgs. Ética na Pesquisa em Saúde. $1^{\mathrm{a}}$ ed. Brasília, DF: Letras Livres e Editora UNB; 2007. p. 31-54

10. Brennan TA. Proposed revisions to the Declaration of Helsinki - Will they weaken the ethical principles underlying human research? N Eng J Med 1999; 341:527-530.

11. Diniz D, Corrêa M. Declaração de Helsinki: relativismo e vulnerabilidade. Cad Saude Publica 2001; 17:679-688.

12. Council for International Organizations of Medical Sciences (CIOMS). International Ethical Guidelines for Biomedical Research Involving Human Subjects. [Internet] [Cited 2012 August 2]. Available from: http://www.cioms. ch/images/stories/CIOMS/guidelines/guidelines nov 2002 blurb.htm.

13. Lackey DP. Clinical research in developing countries: recent moral arguments. Cad Saude Publica 2002; 18:1455-1461.

14. Ellenberg SS, Fleming TR, DeMets DL. Data monitoring committee interactions with other trial components or related groups. In: Ellenberg SS, Fleming TR, DeMets DL, editors. Data monitoring committees in clinical Trials. Chichester, England: John Willey \& Sons; 2003. p. 105-117.

15. DAMOCLES Study Group. A proposed charter for clinical trials data monitoring committees: helping them to do their job well. Lancet $2005 ; 365: 711-722$.

16. World Health Organization (WHO). Operational Guidelines for Ethics Committees that review biomedical research. TRD/PRD/Ethics/2000.1. Geneva: WHO; 2000 .

17. World Health Organization (WHO). Surveying and evaluating ethical review practices. A complementary guideline to the Operational Guidelines for Ethics Committees that review biomedical research. TRD/PRD/Ethics/2002.1. Geneva: WHO; 2001.

18. International committee of medical journal editors. Uniform requirements for manuscripts submitted to biomedical journals: writing and editing for biomedical publication. [Internet] [Cited 2012 August 2]. Available from: http://www.icmje. org/urm_main.html/.

19. Newell JN, Baral SC, Pande SB, Bam DS, Malla P. Family-member DOTS and community DOTS for tuberculosis control in Nepal: cluster-randomised controlled trial. Lancet 2006; 367:903-909. 